\title{
Bacterial lipopolysaccharides variably modulate in vitro biofilm formation of Candida species
}

\begin{abstract}
Correspondence
L. P. Samaranayake

lakshman@hkucc.hku.hk
\end{abstract}

Received 28 April 2010

Accepted 21 June 2010

\author{
H. M. H. N. Bandara, ${ }^{1}$ O. L. T. Lam, ${ }^{2}$ R. M. Watt, ${ }^{1}$ L. J. Jin ${ }^{3}$ \\ and L. P. Samaranayake ${ }^{1}$
}
${ }^{1}$ Oral Biosciences, Faculty of Dentistry, University of Hong Kong, 5/F Prince Philip Dental Hospital, 34 Hospital Road, Sai Ying Pun, Hong Kong SAR
${ }^{2}$ Oral Rehabilitation, Faculty of Dentistry, University of Hong Kong, Sai Ying Pun, Hong Kong SAR
${ }^{3}$ Periodontology, Faculty of Dentistry, University of Hong Kong, Sai Ying Pun, Hong Kong SAR

The objective of this study was to evaluate the effect of the bacterial endotoxin LPS on Candida biofilm formation in vitro. The effect of the LPS of Pseudomonas aeruginosa, Klebsiella pneumoniae, Serratia marcescens and Salmonella typhimurium on six different species of Candida, comprising Candida albicans ATCC 90028, Candida glabrata ATCC 90030, Candida krusei ATCC 6258, Candida tropicalis ATCC 13803, Candida parapsilosis ATCC 22019 and Candida dubliniensis MYA 646, was studied using a standard biofilm assay. The metabolic activity of in vitro Candida biofilms treated with LPS at $90 \mathrm{~min}, 24 \mathrm{~h}$ and $48 \mathrm{~h}$ was quantified by XTT reduction assay. Viable biofilm-forming cells were qualitatively analysed using confocal laser scanning microscopy (CLSM), while scanning electron microscopy (SEM) was employed to visualize the biofilm structure. Initially, adhesion of C. albicans was significantly stimulated by Pseudomonas and Klebsiella LPS. A significant inhibition of Candida adhesion was noted for the following combinations: C. glabrata with Pseudomonas LPS, C. tropicalis with Serratia LPS, and C. glabrata, C. parapsilosis or C. dubliniensis with Salmonella LPS $(P<0.05)$. After 24 h of incubation, a significant stimulation of initial colonization was noted for the following combinations: C. albicans/C. glabrata with Klebsiella LPS, C. glabrata/C. tropicalis/C. krusei with Salmonella LPS. In contrast, a significant inhibition of biofilm formation was observed in C. glabrata/C. dubliniensis/C. krusei with Pseudomonas LPS, C. krusei with Serratia LPS, C. dubliniensis with Klebsiella LPS and C. parapsilosis/C. dubliniensis /C. krusei with Salmonella LPS $(P<0.05)$. On further incubation for $48 \mathrm{~h}$, a significant enhancement of biofilm maturation was noted for the following combinations: C. glabrata/C. tropicalis with Serratia LPS, C. dubliniensis with Klebsiella LPS and C. glabrata with Salmonella LPS, and a significant retardation was noted for C. parapsilosis/C. dubliniensis/C. krusei with Pseudomonas LPS, C. tropicalis with Serratia LPS, C. glabrata/C. parapsilosis/C. dubliniensis with Klebsiella LPS and C. dubliniensis with Salmonella LPS $(P<0.05)$. These findings were confirmed by SEM and CLSM analyses. In general, the inhibition of the biofilm development of LPS-treated Candida spp. was accompanied by a scanty architecture with a reduced numbers of cells compared with the profuse and densely colonized control biofilms. These data are indicative that bacterial LPSs modulate in vitro Candida biofilm formation in a species-specific and time-dependent manner. The clinical and the biological relevance of these findings have yet to be explored.

\section{INTRODUCTION}

Most micro-organisms exist in nature as complex communities attached to surfaces rather than as isolated free-floating

\footnotetext{
Abbreviations: CLSM, confocal laser scanning microscopy; SEM, scanning electron microscopy.

Tables of XTT reduction analysis data are available as supplementary material with the online version of this paper.
}

planktonic organisms. These structurally and functionally complex communities, termed biofilms, consist of one or more microbial species encased in an extracellular polymeric substance, and attached to one another or to a solid surface (Samaranayake \& MacFarlane, 1990). Biofilms, in comparison to their planktonic counterparts, are resistant to antimicrobials (Mah \& O'Toole, 2001; O'Toole et al., 2000), and are thought to be involved in up to $65 \%$ of all human microbial infections (McLean et al., 2004). 
Biofilms quite frequently comprise multiple species of either bacteria alone (monospecies) or bacteria together with fungi such as Candida (multispecies) (Bandara et al., 2009; Nobile \& Mitchell, 2007). The dimorphic fungus Candida resides in human oral cavities, the vagina, the gastrointestinal tract, on skin and on other mucosal surfaces as a commensal organism (Jenkinson \& Douglas, 2002; Samaranayake \& MacFarlane, 1990). Though the genus Candida comprises more than 150 yeast species, only a few, Candida albicans, Candida tropicalis, Candida parapsilosis, Candida krusei, Candida kefyr, Candida glabrata and Candida guilliermondii, are recognized as medically important pathogens (Odds, 1988). C. albicans is the fourth leading cause of nosocomial bloodstream infection in patients in medical intensive care units (Pittet \& Wenzel, 1995; Richards et al., 1999), and causes superficial to deep infections in neonates, diabetics and immunosuppressed patients (Eubanks et al., 1993; Pfaller, 1996).

LPS, a bacterial endotoxin, is an amphiphilic molecule, which is heterogeneous both in size and composition (Aurell \& Wistrom, 1998). It is an outer cell wall component of Gram-negative bacteria that initiates septic shock in humans and animals through its proinflammatory and immunomodulatory properties (Aurell \& Wistrom, 1998; Morrison \& Ryan, 1987; Rietschel et al., 1982). LPS also induces a variety of responses in monocytes and polymorphonuclear cells, which may play a role in the mechanism of endotoxic shock (Michalek et al., 1980; Tracey et al., 1987); other manifestations of LPS may play a role in the pathophysiology of infections (Morrison \& Ryan, 1987; Young, 1985).

The interactions between LPS and Candida have been sparsely investigated. In previous studies, Henry-Stanley et al. (2003) demonstrated that Escherichia coli LPS enhances the lethality of parenterally administered $C$. albicans. Palma et al. (1992), in an in vitro study, showed that LPS from E. coli, Serratia marcescens and Salmonella typhimurium, at doses ranging from 1 to $100 \mathrm{ng} \mathrm{ml}^{-1}$, strongly enhanced the inhibitory response of human polymorphonuclear leukocytes against the growth of $C$. albicans. Furthermore, the number of yeasts ingested per neutrophil, as well as the number of neutrophils capable of ingesting fungal cells, was markedly increased as a result of LPS exposure (Palma et al., 1992). Our group recently demonstrated, for the first time, the direct modulatory effect of E. coli LPS on different stages of Candida biofilm formation. E. coli LPS significantly suppressed the initial colonization of Candida dubliniensis, while it enhanced the initial colonization of $C$. tropicalis and maturation of $C$. parapsilosis biofilms (Bandara et al., 2009).

Numerous in vivo and in vitro studies have shown that various bacteria and Candida have complex interactions in mixed-species biofilms. This suggests that bacterial infections may play a modulatory role in Candida infections (Bandara et al., 2009; Hedderwick et al., 2000; Peres-Bota et al., 2004). However, the role of the major Gram-negative bacterial endotoxin, LPS, in mixed infections is yet to be fully unravelled. Though a few studies have been conducted, none have been designed to illustrate the effect of LPSs on the process of biofilm formation of this yeast. Furthermore, the role of different Gram-negative bacterial LPS on candidal biofilms has not been evaluated. Additionally, the majority of the previous studies have focused only on $C$. albicans and, to the best of our knowledge, there are no studies pertaining to non-albicans Candida spp. biofilms.

Hence, the aims of this study were to evaluate the effect of LPS derived from Pseudomonas aeruginosa, Serratia marcescens, Klebsiella pneumoniae and Salmonella typhimurium on six different Candida species, including C. albicans, $C$. glabrata, C. tropicalis, C. parapsilosis, C. dubliniensis and C. krusei, in different stages of biofilm formation, by both quantitative assays (XTT reduction assay), and qualitative evaluations using scanning electron microscopy (SEM) and confocal laser scanning microscopy (CLSM).

\section{METHODS}

Experimental design. The study comprised a series of multiple experiments to evaluate the effect of LPS derived from different Gram-negative bacteria as described below, on Candida spp. biofilm formation, quantitatively with XTT reduction assay, and qualitatively with CLSM and SEM, at three different time intervals, $90 \mathrm{~min}, 24 \mathrm{~h}$ and $48 \mathrm{~h}$. These time intervals corresponded to the adhesion phase, initial colonization phase and the maturation phase of Candida biofilms, respectively. These latter definitions and terminology are used consistently in the following sections of the paper.

Micro-organisms. The following reference laboratory strains of Candida were used: C. albicans ATCC 90028, C. glabrata ATCC 90030, C. tropicalis ATCC 13803, C. parapsilosis ATCC 22019, C. krusei ATCC 6258 and C. dubliniensis MYA 646. The identity of each organism was confirmed with the commercially available API $32 \mathrm{C}$ identification system (bioMérieux). Prior to experiments, all isolates were stored in multiple aliquots at $-20{ }^{\circ} \mathrm{C}$, after confirming their purity.

LPS. LPSs purified by phenol extraction from K. pneumoniae ATCC 15380 (catalogue no. L4268), Serratia marcescens ATCC 21639 (catalogue no. L6136), Salmonella typhimurium ATCC 7823 (catalogue no. L6511), and P. aeruginosa ATCC 27316 (catalogue no. L9143) were purchased in the form of lyophilized powder from Sigma Aldrich and stored $2-8{ }^{\circ} \mathrm{C}$ until use.

Growth media. Sabouraud dextrose agar and yeast nitrogen base (YNB) solution supplemented with $100 \mathrm{mM}$ glucose were used for culturing Candida species.

Microbial inocula. Prior to each experiment, Candida spp. were subcultured on Sabouraud dextrose agar for $18 \mathrm{~h}$ at $37^{\circ} \mathrm{C}$. A loopful of this overnight Candida growth was inoculated into YNB medium and incubated for $18 \mathrm{~h}$ in an orbital shaker ( 75 r.p.m.) at $37{ }^{\circ} \mathrm{C}$. The resultant growth was harvested, washed twice in PBS (pH 7.2, $\mathrm{Ca}^{2+}$ and $\mathrm{Mg}^{2+}$ free; Sigma) and resuspended. PBS without calcium and magnesium ions was used to wash and rinse suspended cells as these ions facilitate cell binding and clumping, which will mask the effect of LPS on Candida during biofilm development. Concentrations of Candida spp. were adjusted $1 \times 10^{7}$ cells $\mathrm{ml}^{-1}$ by spectrophotometry and confirmed by haemocytometric counting. 
Biofilm formation. Candida biofilms were prepared as described by Jin et al. (2004), with some modifications. Commercially available presterilized, polystyrene, flat bottom 96-well microtitre plates (Iwaki) were used. At first, $100 \mu \mathrm{l}$ standard cell suspension of Candida spp. $\left(10^{7}\right.$ organisms $\left.\mathrm{ml}^{-1}\right)$ was prepared and transferred into the wells of a microtitre plate, and the plate incubated for $1.5 \mathrm{~h}$ at $37{ }^{\circ} \mathrm{C}$ in an orbital shaker at 75 r.p.m. to promote microbial adherence to the surface of the wells. After the initial adhesion phase, the cell suspensions were aspirated and each well was washed twice with PBS to remove loosely adherent cells. A total of $200 \mu \mathrm{l}$ YNB was transferred to each well and the plate reincubated for 24 and $48 \mathrm{~h}$, and the wells washed twice and three times at the respective time intervals with PBS to eliminate traces of YNB. Thus, the effect of bacterial LPS on Candida was studied at $90 \mathrm{~min}, 24 \mathrm{~h}$ and $48 \mathrm{~h}$ time intervals.

Quantitative analyses - evaluation of LPS impact using XTT reduction assay. Freshly obtained LPS $\left(1 \mathrm{mg} \mathrm{ml}^{-1}\right.$; Sigma Aldrich) and YNB supplemented with $100 \mathrm{mM}$ glucose were prepared to yield a final dilution of LPS ranging from $100 \mu \mathrm{g} \mathrm{ml}^{-1}$ to $1 \mathrm{ng} \mathrm{ml}^{-1}$. LPS was replaced with sterile PBS in the control samples. Candida biofilms were formed in three different 96-well plates to evaluate the effect of LPS at $90 \mathrm{~min}, 24 \mathrm{~h}$ and $48 \mathrm{~h}$ (Fig. 1) as described below.

First, a Candida suspension (100 $\mu \mathrm{l}$ each) resuspended in test and control media (see above) was dispersed into selected wells of a 96well plate at time 0 and incubated in an orbital shaker (75 r.p.m.) at $37{ }^{\circ} \mathrm{C}$ for $90 \mathrm{~min}$ for the adhesion assay. In the second assay, $200 \mu \mathrm{l}$ test and control media were added at $90 \mathrm{~min}$ to a different 96 -well plate with pre-adherent Candida and incubated for $24 \mathrm{~h}$ for the initial colonization study. Finally, in the third assay, the latter procedure was repeated at $24 \mathrm{~h}$ for initially colonized Candida, in a different 96-well plate, and incubated for further $24 \mathrm{~h}$ for the maturation phase study.

At the end of incubation, the supernatant was removed and wells washed twice with sterile PBS to remove loosely adherent cells. A standard XTT reduction assay was performed thereafter as described by Jin et al. (2004) to measure the metabolic activity of the biofilms. In brief, commercially available XTT powder (Sigma) was dissolved in PBS at a final concentration of $1 \mathrm{mg} \mathrm{ml}^{-1}$, then the solution was filter-sterilized $\left(0.22 \mu \mathrm{m}\right.$ pore size filter) and stored at $-70{ }^{\circ} \mathrm{C}$. Freshly prepared $0.4 \mathrm{mM}$ menadione solution was used for the assay. XTT solution was thawed and mixed with menadione solution at 20:1 (v/v) immediately before the assay. Thereafter, $158 \mu \mathrm{l}$ PBS, $40 \mu \mathrm{l}$ XTT and $2 \mu \mathrm{l}$ menadione solution were added into each well containing prewashed biofilms and incubated in the dark for $3 \mathrm{~h}$ at $37^{\circ} \mathrm{C}$. The colour change was measured by absorbance with a microtitre plate reader (Spectra Max 340 tunable microplate reader; Molecular devices) at $492 \mathrm{~nm}$. All assays were carried out in triplicate on three different occasions.

Qualitative analyses. CLSM (Jin et al., 2005) and SEM were used to observe the ultrastructure of test and control Candida biofilms (Fig. 1).
CLSM. Commercially available presterilized flat-bottomed six-well plates (Iwaki) and presterilized plastic coverslips (Thermanox; Nulge Nunc International) (Ramage et al., 2001) were used to prepare biofilms as described above. Presterilized coverslips were placed in the wells of a six-well plate, Candida suspensions added and the plate incubated for $90 \mathrm{~min}$ (the adhesion phase) in an orbital shaker (75 r.p.m.) at $37^{\circ} \mathrm{C}$. Thereafter, the supernatant was removed, washed twice with PBS, fresh YNB added and incubated for $24 \mathrm{~h}$ (initial colonization) or $48 \mathrm{~h}$ (maturation) under the same environmental conditions. At the end of each time interval, the prewashed coverslips were stained with LIVE/DEAD stain (LIVE/DEAD BacLight Bacterial viability kit; Invitrogen) (Jin et al., 2005). The biofilm was then analysed by fluorescent microscopy (using a confocal laser scanning microscope).

SEM. For SEM, we prepared Candida species biofilms (both control and LPS treated) on custom-made, tissue culture-treated, polystyrene coverslips (prepared in six-well plates) as described above. At $90 \mathrm{~min}$, $24 \mathrm{~h}$ and $48 \mathrm{~h}$, selected coverslips were removed from the wells, washed twice with PBS and placed in $1 \%$ osmium tetroxide for $1 \mathrm{~h}$. Samples were subsequently washed in distilled water, dehydrated in a series of ethanol washes ( $70 \%$ for $10 \mathrm{~min}, 95 \%$ for $10 \mathrm{~min}$ and $100 \%$ for $20 \mathrm{~min}$ ), and air-dried in a desiccator prior to sputter coating with gold. Then the specimens were mounted on aluminium stubs, with copper tape, and coated with gold under low-pressure with an ion sputter coater (JFC1 100; JEOL). The surface topographies of the biofilm were visualized with a scanning electron microscope (Philip $\mathrm{XL} 30 \mathrm{CP}$ ) in high-vacuum mode at $10 \mathrm{kV}$, and the images processed.

Statistical analysis. Statistical analysis was performed using SPSS software (version 16.0). The Mann-Whitney U-test was performed to compare significant differences between the control and each test sample of the candidal biofilm. Data from all Candida spp. at different time points $(90 \mathrm{~min}, 24 \mathrm{~h}$ and $48 \mathrm{~h}$ ) were pooled and evaluated against a concentration gradient of different LPS using 2sample $t$-test. A similar statistical test was used to analyse pooled data from all LPS experiments at a concentration gradient against different Candida spp. at different time points (90 min, $24 \mathrm{~h}$ and $48 \mathrm{~h}$ ). A $P$ value of $<0.05$ was considered statistically significant.

\section{RESULTS}

\section{Effect of E. coli LPS on Candida biofilms (XTT reduction assay)}

In the early biofilm development phase, after $90 \mathrm{~min}$ of incubation, some of the LPS-treated Candida species demonstrated significant changes in metabolic activity
Candida spp. biofilm formation

1 $90 \mathrm{~min} 24 \mathrm{~h}$

$24 \mathrm{~h}$

$48 \mathrm{~h}$
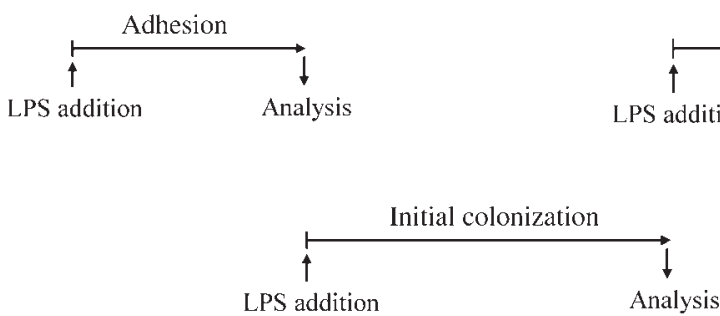

Fig. 1. Summary of the experimental design. Candida biofilm formation was evaluated at three different time intervals: 0-90 min (adhesion phase), 90 min-24 h (initial colonization phase) and 24-48 h (maturation phase). At the commencement of each time interval, varying concentrations of LPS were added to Candida biofilms in 96-well plates; at the end of each phase, quantitative and qualitative analyses were performed using the XTT reduction assay, and CLSM and SEM, respectively. 
compared with their controls. With Pseudomonas LPS, C. albicans showed significant enhancement of its metabolic activity at $10 \mu \mathrm{g} \mathrm{ml}^{-1}$ and in contrast, C. glabrata was significantly inhibited at $100 \mu \mathrm{g} \mathrm{ml}^{-1}, 10 \mu \mathrm{g} \mathrm{ml}^{-1}, 100 \mathrm{ng} \mathrm{ml}^{-1}$ and $10 \mathrm{ng} \mathrm{ml}^{-1}$. Adhesion of the other four Candida species were unaffected with Pseudomonas LPS treatment. With Serratia LPS, C. tropicalis was the only species affected out of all six Candida species, and demonstrated significantly reduced XTT readings at the concentrations of $100 \mu \mathrm{g} \mathrm{ml}^{-1}$, $10 \mu \mathrm{g} \mathrm{ml}^{-1}$ and $1 \mu \mathrm{g} \mathrm{ml}^{-1}$. With Salmonella LPS, a similar effect was observed in the adhesion phase of $C$. glabrata at $100 \mu \mathrm{g} \mathrm{ml}^{-1}$ and $10 \mu \mathrm{g} \mathrm{ml}^{-1}$, C. parapsilosis at $100 \mu \mathrm{g} \mathrm{ml}^{-1}$ and C. dubliniensis at $10 \mu \mathrm{g} \mathrm{ml}^{-1}$, while the adhesion of $C$. albicans, C. tropicalis and C. krusei remained unaffected. In contrast, Klebsiella LPS significantly increased the metabolic activity of only C. albicans early biofilms at $10 \mu \mathrm{g} \mathrm{ml}^{-1}$ concentration (Table 1, Supplementary Tables S1, S2, S3, S4, S5 and S6 available with the online journal).

However, after prolonged incubation, for $24 \mathrm{~h}$, beyond the initial colonization phase of the biofilms, significant variations in XTT readings were seen in all Candida spp. Thus, upon Pseudomonas LPS treatment, C. glabrata at $100 \mu \mathrm{g} \mathrm{ml}^{-1}$, $10 \mu \mathrm{g} \mathrm{ml}^{-1}$ and $1 \mathrm{ng} \mathrm{ml}^{-1}$, and C. dubliniensis and C. krusei at $100 \mu \mathrm{g} \mathrm{ml}^{-1}$ showed significantly reduced XTT values. $C$. albicans, C. tropicalis and C. parapsilosis showed uninterrupted colonization at this stage. A stimulatory effect was detected in C. krusei biofilms treated with $10 \mu \mathrm{g}$ Serratia LPS $\mathrm{ml}^{-1}$ and the colonization of remaining five Candida species was unaffected. C. dubliniensis treated with $100 \mu \mathrm{g}$ Klebsiella LPS $\mathrm{ml}^{-1}$ also demonstrated a significant reduction in XTT readings. In contrast, Klebsiella LPS-treated biofilms of $C$. albicans at $100 \mu \mathrm{g} \mathrm{ml}^{-1}$, and C. glabrata at $10 \mu \mathrm{g} \mathrm{ml}^{-1}$ and $1 \mu \mathrm{g} \mathrm{ml}^{-1}$, showed a significant stimulation of metabolic activity, whilst C. tropicalis, C. parapsilosis and C. krusei showed normal biofilm growth. Salmonella LPS also had a similar stimulatory effect on C. glabrata at $100 \mathrm{ng} \mathrm{ml}^{-1}$, on C. tropicalis at $100 \mu \mathrm{g} \mathrm{ml}^{-1}$ and $10 \mathrm{ng} \mathrm{ml}^{-1}$, and on C. krusei at $1 \mathrm{ng} \mathrm{ml}^{-1}$. However, Salmonella LPS significantly lowered the metabolic activity of C. parapsilosis at $100 \mathrm{ng} \mathrm{ml}^{-1}$, and C. dubliniensis and C. krusei at $100 \mu \mathrm{g} \mathrm{ml}^{-1}$. C. albicans could withstand the effects of Salmonella LPS at this stage (Table 1).

On further incubation for $48 \mathrm{~h}$, Pseudomonas LPS demonstrated a significant suppression of metabolic activities of $C$. parapsilosis (at $100 \mu \mathrm{g} \mathrm{ml}^{-1}, 10 \mu \mathrm{g} \mathrm{ml}^{-1}$ and $100 \mathrm{ng} \mathrm{ml}^{-1}$ ), C. dubliniensis (at $100 \mu \mathrm{g} \mathrm{ml}^{-1}$ ) and C. krusei (at $100 \mu \mathrm{g} \mathrm{ml}^{-1}$, $10 \mu \mathrm{g} \mathrm{ml}^{-1} 100 \mathrm{ng} \mathrm{ml}^{-1}$ and $1 \mathrm{ng} \mathrm{ml}^{-1}$ ); although, the maturation of C. albicans, C. glabrata and C. tropicalis biofilms were unaffected in the presence of Pseudomonas LPS. In contrast, when treated with Serratia LPS, an elevated metabolic activity could be observed in C. glabrata (at $100 \mu \mathrm{g} \mathrm{ml}^{-1}$ and $10 \mathrm{ng} \mathrm{ml}^{-1}$ ), and C. tropicalis (at $100 \mu \mathrm{g} \mathrm{ml}^{-1}, 10 \mu \mathrm{g} \mathrm{ml}^{-1}$ and $1 \mu \mathrm{g} \mathrm{m}^{-1}$ ). Conversely, C. parapsilosis showed the opposite response at $100 \mu \mathrm{g} \mathrm{ml}^{-1}$ though C. albicans, C. dubliniensis and C. krusei biofilms were unaffected. When treated with Klebsiella LPS, C. glabrata (at $10 \mu \mathrm{g} \mathrm{ml}^{-1}$ ), and C. dubliniensis (at $100 \mu \mathrm{g} \mathrm{ml}^{-1}$ ) demonstrated significantly low XTT readings compared to the controls, though a significantly higher reading was noted with $C$. parapsilosis (at $100 \mu \mathrm{g} \mathrm{ml}^{-1}$ and $10 \mu \mathrm{g} \mathrm{ml}{ }^{-1}$ ) and C. dubliniensis (at $100 \mathrm{ng} \mathrm{ml}^{-1}$ and $10 \mathrm{ng} \mathrm{ml}^{-1}$ ). The remaining three Candida species tested were unaffected. A significant increase in the metabolic activity of C. glabrata was observed after treatment with Salmonella LPS (at $100 \mu \mathrm{g} \mathrm{ml}^{-1}, 1 \mu \mathrm{g} \mathrm{ml}^{-1}$ and $100 \mathrm{ng} \mathrm{ml}^{-1}$ concentrations). In contrast, a lower XTT reading was detected in $C$. dubliniensis biofilms treated with $100 \mu \mathrm{g}$ Salmonella LPS $\mathrm{ml}^{-1}$. C. albicans, C. parapsilosis and C. dubliniensis biofilms showed normal maturation despite the treatment with Salmonella LPS (Table 1).

We further analysed the pooled data from the foregoing experiments due to the high variability of the results. This was performed in two ways: first, the data from all Candida species against LPS of different Gram-negative bacterial species were pooled and analysed; second, the data from all bacterial LPS against six different Candida spp. were pooled and analysed.

In the first analysis, after 90 min of incubation (adhesion phase) Salmonella LPS at $100 \mu \mathrm{g} \mathrm{ml}^{-1}$ and $10 \mu \mathrm{g} \mathrm{ml}^{-1}$ significantly suppressed the metabolic activity of Candida spp. in general. However, the remaining types of LPS tested, at any given concentration, had no significant effects on the adhesion $(90 \mathrm{~min})$, initial colonization $(24 \mathrm{~h})$ or biofilm maturation ( $48 \mathrm{~h}$ ) of Candida species.

Furthermore, when the data from all the tests of bacterial LPS against six different Candida spp. were pooled and analysed, we noted that the metabolic activity of the $C$. albicans $24 \mathrm{~h}$ biofilm was significantly higher when treated with $100 \mu \mathrm{g}$ LPS $\mathrm{ml}^{-1}$. In contrast, at a similar concentration of LPS, C. dubliniensis $24 \mathrm{~h}$ and $48 \mathrm{~h}$ biofilms, and C. krusei $24 \mathrm{~h}$ biofilm demonstrated a significant reduction in the metabolic activity. C. glabrata, C. tropicalis and C. parapsilosis in all three stages of biofilm formation could withstand any given concentration of LPS without any significant effect.

\section{CLSM}

CLSM with LIVE/DEAD stain showed an increase in C. albicans blastospores after incubation with Pseudomonas LPS for $90 \mathrm{~min}$, confirming the data from XTT reduction assay (Fig. 2a, b). Furthermore, LPS-treated C. albicans were uniform in size, clumped and showed a higher proportion of budding yeasts compared to its control (Fig. 2b), whilst a considerable number of dead yeasts were visible among scattered C. albicans in the control (Fig. 2a).

After $24 \mathrm{~h}$ of incubation (initial colonization phase), growth suppression of C. krusei by Salmonella LPS was clearly seen by CLSM with LIVE/DEAD stain, confirming the XTT reduction assay data (Fig. 2c, d). Thus a few, scant C. krusei blastospores were scattered in the microscopic field. Yeasts were small in size and few showed budding morphology. Some dead and degrading cells were also present in sparsely visible matrix (Fig. $2 \mathrm{~d}$ ). In contrast, its 
Table 1. Percentage modulation of biofilm growth of six different Candida spp. by six different concentrations of LPS from four different bacteria at varying time intervals

Biofilm activity was determined by XTT reduction assay at $90 \mathrm{~min}, 24 \mathrm{~h}$ and $48 \mathrm{~h}$ biofilm formation. The Mann-Whitney $\mathrm{U}$ test was performed and a $P$ value $<0.05$ was considered as statistically significant. Positive percentages indicate LPS concentrations showing significant biofilm stimulation. Negative percentages indicate LPS concentrations showing a significant suppression. Due to the complexity and multitude of data, only the combinations that showed significant findings are indicated in the table.

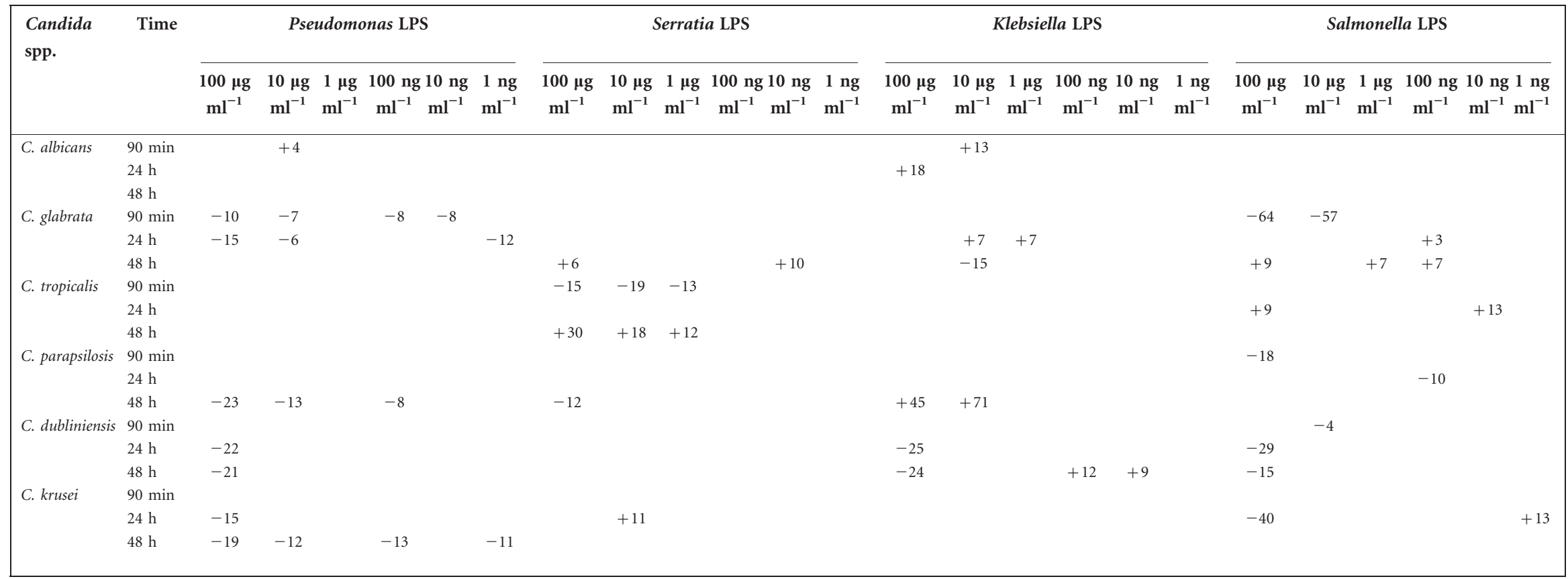


(a)

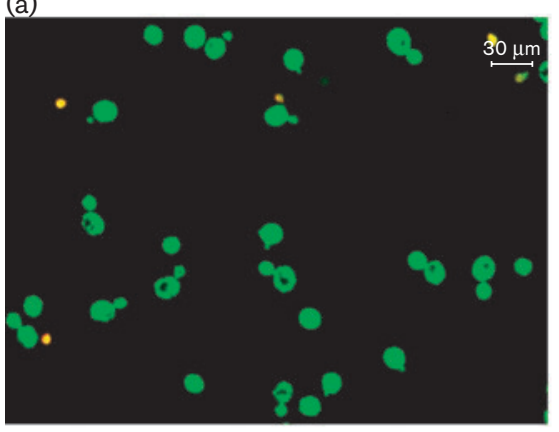

(c)

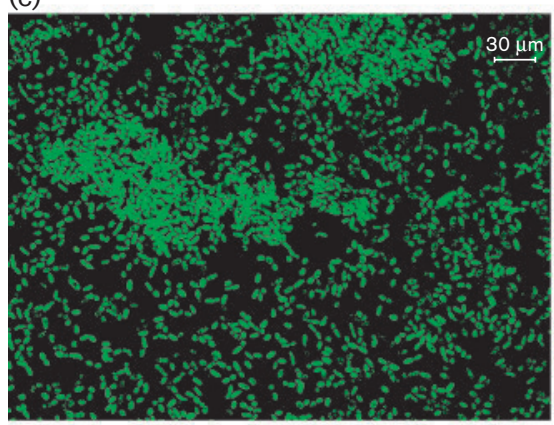

(e)

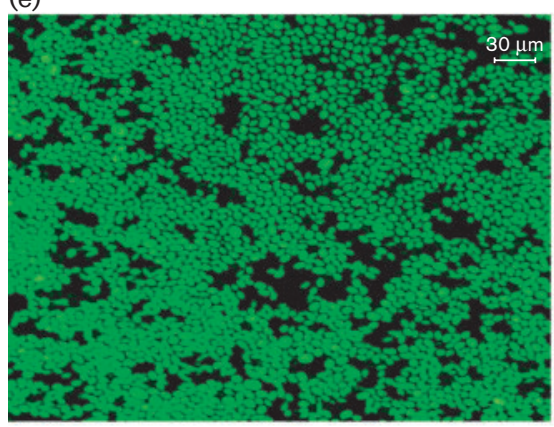

(g)

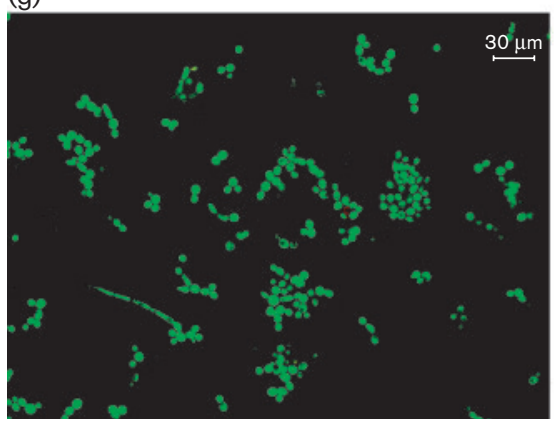

(b)

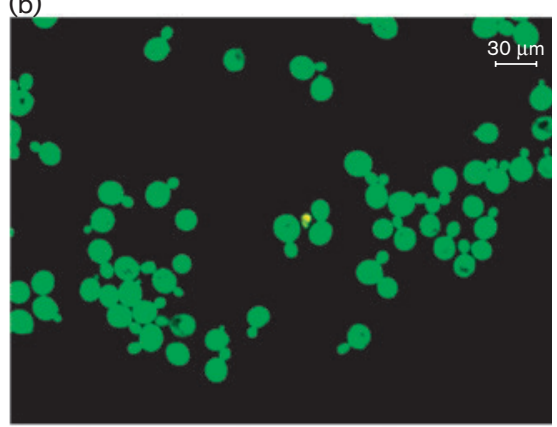

(d)

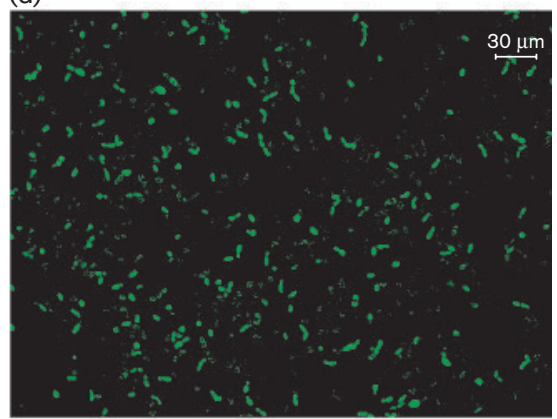

(f)

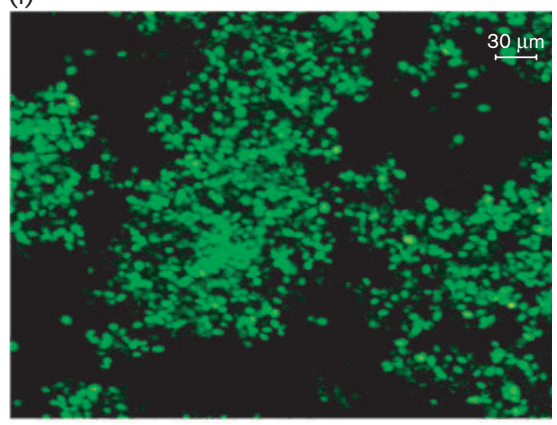

(h)

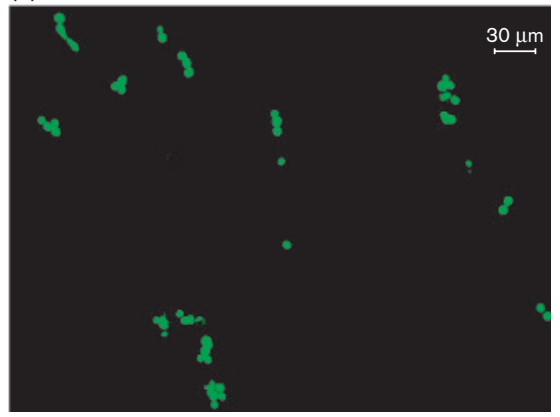

Fig. 2. CLSM images of Candida spp. biofilms treated with Gram-negative bacterial LPS (magnification $\times 40$ ) (stained using a LIVE/DEAD BacLight bacterial viability kit; Invitrogen). Live cells are stained in green and dead cells in red. (a, b) Adhesion of $C$. albicans at 90 min: (a) control, (b) treated with Pseudomonas LPS $\left(10 \mu \mathrm{g} \mathrm{m}^{-1}\right)$. Note the enhancement of C. albicans adhesion due to Pseudomonas LPS in comparison to its untreated counterpart. (c, d) Initial colonization of $C$. krusei at $24 \mathrm{~h}$ : (c) control, (d) treated with Salmonella LPS $\left(100 \mu \mathrm{g} \mathrm{m}{ }^{-1}\right)$. Note the profuse, healthy control biofilm of C. krusei, compared with the Salmonella LPS-treated biofilm with scanty growth. (e, f) Initial colonization of C. glabrata at 24 h: (e) control, (f) treated with Pseudomonas LPS $\left(100 \mu \mathrm{g} \mathrm{ml}^{-1}\right)$. Note the suppression of C. glabrata growth in the Pseudomonas LPS-treated sample. (g, h) Maturation of C. dubliniensis at $48 \mathrm{~h}$ : $(\mathrm{g})$ control; $(\mathrm{h})$ treated with Salmonella LPS $\left(100 \mu \mathrm{g} \mathrm{ml}^{-1}\right)$. Note the suppression of C. dubliniensis by Salmonella LPS. 
control counterpart showed a higher proportion of uniform and elongated C. krusei blastospores arranged in clusters (Fig. 2c). Similarly, at the same time point (Fig. 2e, $\mathrm{f}$, groups of irregular shaped blastospores of C. glabrata were visible in the Pseudomonas LPS-treated $\left(100 \mu \mathrm{g} \mathrm{ml}^{-1}\right)$ sample (Fig. 2f), while the C. glabrata control was densely populated with uniform yeasts (Fig. 2e).

After $48 \mathrm{~h}$ of incubation (maturation phase), sparsely distributed C. dubliniensis blastospores were noted in biofilms treated with Salmonella LPS, reconfirming the foregoing quantitative (XTT) findings (Fig. 2g, h). Yeasts were anisotropic, with some cellular debris in the background (Fig. 2h) compared to the control where high numbers of $C$. dubliniensis yeast forms and some hyphae were evident (Fig. 2g).

\section{SEM}

After 90 min of incubation (adhesion phase), increased numbers of blastospores adherent to the polystyrene surface were noted in $C$. albicans treated with Pseudomonas LPS $\left(100 \mu \mathrm{g} \mathrm{ml}^{-1}\right)$ (Fig. 3a, b). Yeasts of varying sizes were noted and some with irregular cell profiles appeared to be degrading (Fig. 3b). In contrast, the C. albicans control had lesser numbers of yeasts of more uniform size, and healthier than those treated with LPS (Fig. 3a)

After $24 \mathrm{~h}$ of incubation, the C. dubliniensis control characteristically exhibited a profuse, dense growth; although, the extracellular matrix was scarcely visible under SEM. Yeasts were uniform in appearance (Fig. 3c). However, C. dubliniensis treated with Pseudomonas LPS $\left(100 \mu \mathrm{g} \mathrm{ml}^{-1}\right)$ showed a scanty biofilm with reduced numbers of cell layers and reduced cell regularity. Yeasts were different in size and shape (Fig. 3d).

After $48 \mathrm{~h}$ of incubation for biofilm maturation, C. krusei biofilm controls demonstrated elongated, more uniform yeasts with a substantial amount of extracellular matrix. Some dead and degrading cells could also be observed under SEM (Fig. 3e). In contrast, C. krusei treated with Pseudomonas LPS $\left(100 \mu \mathrm{g} \mathrm{ml}^{-1}\right)$ showed a scanty biofilm with elongated blastospores and sparse extracellular matrix (Fig. 3f).

\section{DISCUSSION}

Candida is a polymorphic fungus that frequently causes systemic infections in critically ill patients (Rangel-Frausto et al., 1999). Despite advances in antifungal therapy, the upsurge of the incidence of fungaemia in such communities has been noted with concern (Charles et al., 2003; Pappas et al., 2003; Peres-Bota et al., 2004). This ubiquitous yeast is also the fourth most common cause of nosocomial blood-stream infection (Pappas et al., 2003; Rangel-Frausto et al., 1999), and the agent of a significant proportion of crude (61\%) and attributable (49\%) mortality in critically ill patients in North America (Charles et al., 2003).

It was recently estimated that Gram-negative bacteria are involved in half of the cases of sepsis (approx. 500000 cases of sepsis) that occur annually in the USA. Up to $60 \%$ of these cases tend to end in septic shock (Rangel-Frausto, 1999). Gram-negative bacterial LPS, a potent microbial toxin, and its proinflammatory properties are well known to play a critical role in the pathogenesis of such septic shock (Luchi \& Morrison, 2000).

Though C. albicans is the most commonly encountered organism from the genus Candida in clinical specimens, the incidence of non-albicans Candida is rising (Pappas et al., 2003; Peres-Bota et al., 2004; Rangel-Frausto et al., 1999; Snydman, 2003; Wisplinghoff et al., 2004). Furthermore, there is evidence to indicate that bacterial co-infection or superinfection play a critical role in Candida infections (Bandara et al., 2009; Peres-Bota et al., 2004). Some have also reported that Gram-negative bacterial LPS has direct (Bandara et al., 2009) and indirect effects (Henry-Stanley et al., 2003) on Candida biofilm formation, and on candidal infections.

We therefore evaluated the quantitative and qualitative effect of four different Gram-negative bacterial LPSs from $P$. aeruginosa, Serratia marcescens, K. pneumoniae and Salmonella typhimurium on various stages of biofilm development in six different human pathogenic Candida species. Our results are indicative that different LPSs modulate the various stages of biofilm development of specific Candida spp. to varying degrees.

Using the XTT reduction assay, we report here for what is believed to be the first time, the quantitative effects of four different bacterial LPS on six different Candida species at three different time points in biofilm development. When taken individually, each type of LPS tested here had significant effects, either inhibitory or stimulatory, on Candida species. In general, the effect elicited by LPS was time and concentration dependent. Salmonella and Pseudomonas LPS had the strongest overall effect on Candida, while Serratia LPS had the least. The effect of Pseudomonas LPS was mainly inhibitory while, the contrary was true for Salmonella LPS. When the phase of biofilm development was evaluated, Salmonella LPS had the strongest effect on Candida adhesion, being inhibitory in nature and the least effect was noted with Klebsiella LPS. Salmonella LPS effect was mixed, with the strongest effect on the initial candidal colonization ( $24 \mathrm{~h})$ stage. Serratia LPS showed the weakest effect at this stage by only inhibiting C. krusei.

Pseudomonas LPS had the strongest suppressive effect on the maturation phase Candida biofilms. In contrast, Serratia and Salmonella LPS showed the highest, stimulatory, effect on the latter phase of Candida biofilms. Despite these specific variations, Salmonella LPS significantly inhibited the adhesion of Candida spp. at 100 and $10 \mu \mathrm{g} \mathrm{ml}^{-1}$. 
(a)

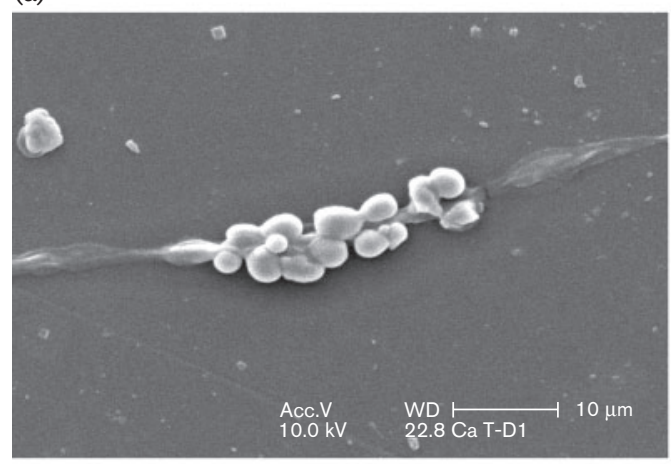

(c)

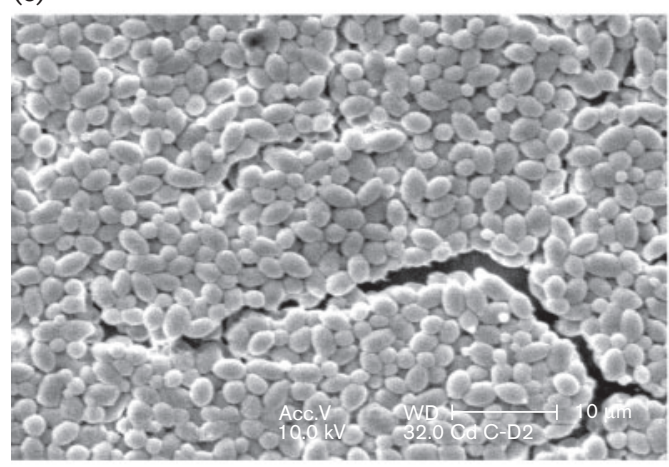

(e)

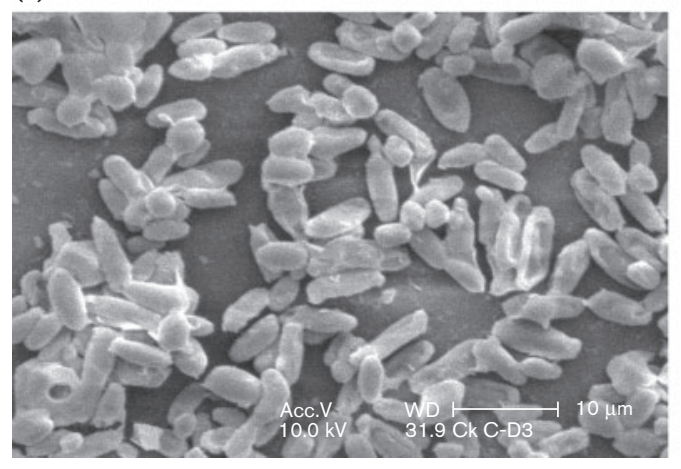

(b)

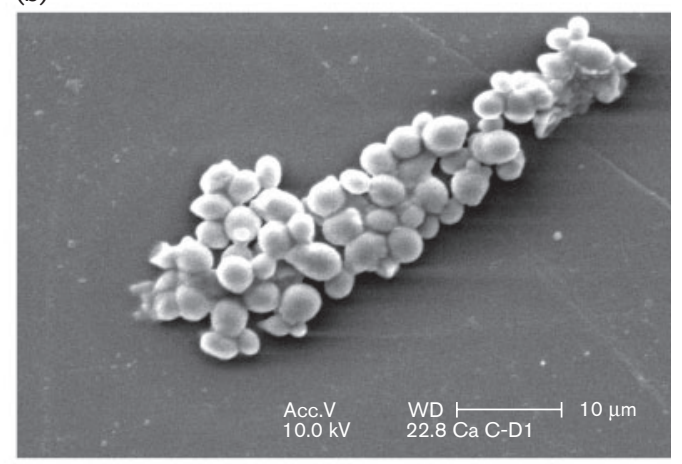

(d)

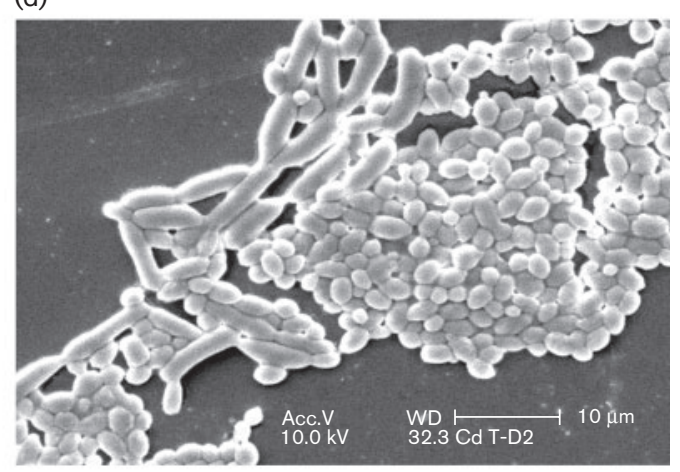

(f)

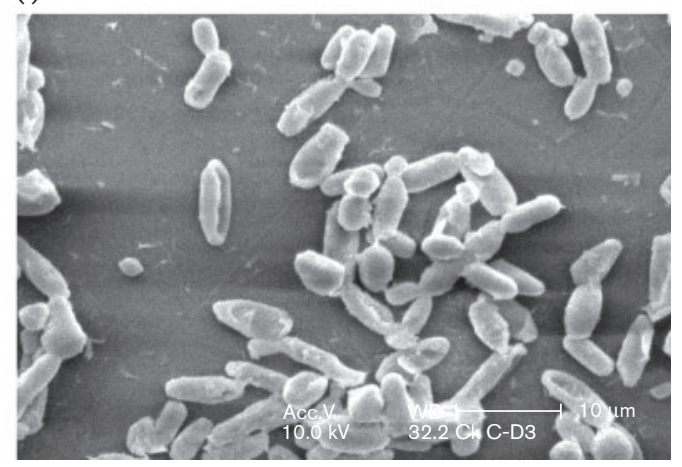

Fig. 3. SEM images of Candida spp. biofilms - control biofilms and biofilms treated with Gram-negative bacterial LPSs (magnification $\times 2000)$. $(\mathrm{a}, \mathrm{b})$ Adhesion of C. albicans (90 min): (a) control, (b) treated with Pseudomonas LPS $\left(10 \mu \mathrm{g} \mathrm{ml}{ }^{-1}\right.$ ). Note the LPS-treated C. albicans cells are heterogeneous in size and more populated in comparison to the control. (c, d) Initial colonization of C. dubliniensis (24 h): (c) control, (d) treated with Pseudomonas LPS $\left(100 \mu \mathrm{g} \mathrm{ml}^{-1}\right)$. Note the LPS-treated C. dubliniensis blastospores are less in number, different in shape and scantily distributed in comparison to the more uniform and densely colonized control. (e, f) Maturation of C. krusei (48 h): (e) control, (f) treated with Pseudomonas LPS (100 $\mu \mathrm{g} \mathrm{ml}^{-1}$ ). Note the LPS-treated C. krusei biofilm is less organized with scattered cells compared to the control.

In general, when the species-specific variations of Candida in response to LPS were considered, C. glabrata was the most susceptible to the LPS effect at any time point of biofilm development; C. albicans and C. tropicalis were the most resistant. At $90 \mathrm{~min}$, the adhesion of C. glabrata was essentially inhibited by LPS. The effect was mixed at $24 \mathrm{~h}$, while it was stimulatory at $48 \mathrm{~h}$. Despite such speciesspecific findings, we noted that the initial colonization of C. albicans was significantly enhanced by $100 \mu \mathrm{g}$ LPS $\mathrm{ml}^{-1}$.
Our results substantiate the concept that Gram-negative bacterial LPSs in general have a direct modulatory effect on Candida biofilm development. The current data also validate the results of our previous study, which showed that E. coli LPS has a modulatory effect on Candida biofilm formation (Bandara et al., 2009). In contrast, Palma et al. (1992) have reported that LPSs of E. coli, Serratia marcescens and Salmonella typhimurium had no direct effect on Candida growth at any concentration. 
The phenotypic variations of LPS could be one important factor that affects its species-specific functional diversity. There is evidence, including the current observations, to support the concept that LPS diversity affects its biological activity. For instance, LPS activity can vary substantially depending upon both the type of the organism and the method of its extraction (Morrison \& Leive, 1975). The presence of outer-membrane proteins in association with LPS (Morrison et al., 1976), the specific subunit composition of LPS (Vukajlovich \& Morrison, 1985) and the presence of $\mathrm{O}$-antigen carbohydrates that are linked to the LPS (Morrison et al., 1987) can all determine the quality of this lipid-carbohydrate complex. Sabra et al. (2003) explained that the phenotypic variations in the relative expression of two chemically distinct types of LPS of $P$. aeruginosa could be an important mechanism that alters surface characteristics in mediating adhesion and survival under extreme conditions (Makin \& Beveridge, 1996; Sabra et al., 2003). It should also be noted that, apart from such chemical properties, the method of LPS preparation may have an effect on its bioactivity and toxicity (Luchi \& Morrison, 2000; Morrison \& Leive, 1975).

Although limited data are available on the effect of LPS in vitro, there are a number of in vivo studies demonstrating the variable effects of LPS on Candida. Burd et al. (1992) noted that when mice were injected with $C$. albicans along with viable $E$. coli or E. coli LPS intraperitoneally, both E. coli as well as LPS enhanced the virulence of C. albicans. In a similar study, Klaerner et al. (1997) also noted that mice injected with E. coli or LPS had increased number of C. albicans in kidney tissue suggesting the promotive effect of LPS on C. albicans (Akagawa et al., 1995). As in the current study, species specificity of LPS appears to influence its outcome in the host. On the contrary, Hess et al. (2009) noted that the tissue burden of C. glabrata in mice was decreased in the presence of E. coli and its LPS. Henry-Stanley et al. (2003) demonstrated that LPS may protect against extraintestinal dissemination of orally inoculated C. albicans by inhibiting the adhesion of yeasts to intestinal epithelium.

It should be borne in mind that proinflammatory and immunomodulatory properties of LPS in vivo are major factors determining its indirect effect on Candida (Morrison \& Ryan, 1987; Rietschel et al., 1982). Palma et al. (1992) noted in an in vitro study that LPS from E. coli, Serratia marcescens and Salmonella typhimurium, at doses from 1 to $100 \mathrm{ng} \mathrm{ml}^{-1}$, strongly enhanced the growth inhibition of $C$. albicans caused by human polymorphonuclear leukocytes.

Ultrastructural views of both LPS-treated and untreated Candida biofilms further confirmed the results of our XTT reduction assays. Generally, when a specific stage of Candida biofilm formation was suppressed by LPS, the biofilm tended to display a rather scanty architecture with a low proportion of budding yeasts, and a preponderance of dead and degrading cells. Candida blastospores were small in size and heterogeneous in shape in comparison to the more uniform, confluent, and relatively well-defined control biofilms. On the contrary, when a specific stage of Candida biofilm formation was stimulated by LPS, the biofilm was richly populated by more isoform blastospores and a large proportion of live and budding Candida, while the controls were devoid of such features.

In clinical terms, our data are indicative that individuals with systemic candidiasis superinfected with Gramnegative bacteria need prompt treatment to eradicate both types of organisms and to neutralize the action of LPS (Akagawa et al., 1995). If indeed some form of LPS does modify the pathogenicity and virulence of Candida, as observed in our study, then it is tempting to suggest that a modified non-toxic form of LPS would be an alternative treatment option for reduction of the incidence of systemic candidiasis in high-risk patients (Henry-Stanley et al., 2003).

In summary, our studies, focused mainly on the effect of various Gram-negative bacterial LPSs on different stages of Candida spp. biofilm development, are indicative that LPS has significant and direct modulatory effects on various stages of Candida biofilm development in a species-specific manner. It is also clear that some Candida species are able to withstand the effect of LPS more than others as the effect of LPS on Candida was specific and diverse. Further work is necessary to explain the molecular mechanisms underlying the species-specific response of Candida against LPS during biofilm formation, and to evaluate the role of LPS as a putative antifungal agent.

\section{ACKNOWLEDGEMENTS}

Authors would like to acknowledge Ms Joyce Yau and Mr Simon Lee for technical help. This study was supported by the University of Hong Kong, grant number CERG HKU 7624/06M.

\section{REFERENCES}

Akagawa, G., Abe, S. \& Yamaguchi, H. (1995). Mortality of Candida albicans-infected mice is facilitated by superinfection of Escherichia coli or administration of its lipopolysaccharide. J Infect Dis 171, 15391544.

Aurell, C. A. \& Wistrom, A. O. (1998). Critical aggregation concentrations of gram-negative bacterial lipopolysaccharides (LPS). Biochem Biophys Res Commun 253, 119-123.

Bandara, H. M., Yau, J. Y., Watt, R. M., Jin, L. J. \& Samaranayake, L. P. (2009). Escherichia coli and its lipopolysaccharide modulate in vitro Candida biofilm formation. J Med Microbiol 58, 1623-1631.

Burd, R. S., Raymond, C. S. \& Dunn, D. L. (1992). Endotoxin promotes synergistic lethality during concurrent Escherichia coli and Candida albicans infection. J Surg Res 52, 537-542.

Charles, P. E., Doise, J. M., Quenot, J. P., Aube, H., Dalle, F., Chavanet, P., Milesi, N., Aho, L. S., Portier, H. \& Blettery, B. (2003). Candidemia in critically ill patients: difference of outcome between medical and surgical patients. Intensive Care Med 29, 2162-2169.

Eubanks, P. J., de Virgilio, C., Klein, S. \& Bongard, F. (1993). Candida sepsis in surgical patients. Am J Surg 166, 617-619. 
Hedderwick, S. A., Lyons, M. J., Liu, M., Vazquez, J. A. \& Kauffman, C. A. (2000). Epidemiology of yeast colonization in the intensive care unit. Eur J Clin Microbiol Infect Dis 19, 663-670.

Henry-Stanley, M. J., Hess, D. J., Erickson, E. A., Garni, R. M. \& Wells, C. L. (2003). Effect of lipopolysaccharide on virulence of intestinal Candida albicans. J Surg Res 113, 42-49.

Hess, D. J., Henry-Stanley, M. J., Bendel, C. M., Zhang, B., Johnson, M. A. \& Wells, C. L. (2009). Escherichia coli and TNF- $\alpha$ modulate macrophage phagocytosis of Candida glabrata. J Surg Res 155, 217224.

Jenkinson, H. F. \& Douglas, L. J. (2002). Interactions between Candida species and bacteria in mixed infections. In Polymicrobial Diseases, pp. 357-373. Edited by K. A. Brogden \& J. M. Guthmiller. Washington, DC: American Society for Microbiology.

Jin, Y., Samaranayake, L. P., Samaranayake, Y. \& Yip, H. K. (2004). Biofilm formation of Candida albicans is variably affected by saliva and dietary sugars. Arch Oral Biol 49, 789-798.

Jin, Y., Zhang, T., Samaranayake, Y. H., Fang, H. H., Yip, H. K. \& Samaranayake, L. P. (2005). The use of new probes and stains for improved assessment of cell viability and extracellular polymeric substances in Candida albicans biofilms. Mycopathologia 159, 353 360 .

Klaerner, H. G., Uknis, M. E., Acton, R. D., Dahlberg, P. S., CarloneJambor, C. \& Dunn, D. L. (1997). Candida albicans and Escherichia coli are synergistic pathogens during experimental microbial peritonitis. J Surg Res 70, 161-165.

Luchi, M. \& Morrison, D. C. (2000). Comparable endotoxic properties of lipopolysaccharides are manifest in diverse clinical isolates of gramnegative bacteria. Infect Immun 68, 1899-1904.

Mah, T. F. \& O'Toole, G. A. (2001). Mechanisms of biofilm resistance to antimicrobial agents. Trends Microbiol 9, 34-39.

Makin, S. A. \& Beveridge, T. J. (1996). Pseudomonas aeruginosa PAO1 ceases to express serotype-specific lipopolysaccharide at $45{ }^{\circ} \mathrm{C}$. J Bacteriol 178, 3350-3352.

McLean, R. J. C., Bates, C. L., Banes, M. B., McGowin, C. L. \& Aron, G. M. (2004). Methods of studying biofilms. In Microbial Biofilms, pp. 279413. Edited by M. Ghannoum \& G. O'Toole. Washington, DC: American Society for Microbiology.

Michalek, S. M., Moore, R. N., McGhee, J. R., Rosenstreich, D. L. \& Mergenhagen, S. E. (1980). The primary role of lymphoreticular cells in the mediation of host responses to bacterial endotoxin. J Infect Dis 141, 55-63.

Morrison, D. C. \& Leive, L. (1975). Fractions of lipopolysaccharide from Escherichia coli O111: B4 prepared by two extraction procedures. J Biol Chem 250, 2911-2919.

Morrison, D. C. \& Ryan, J. L. (1987). Endotoxins and disease mechanisms. Annu Rev Med 38, 417-432.

Morrison, D. C., Betz, S. J. \& Jacobs, D. M. (1976). Isolation of a lipid A bound polypeptide responsible for "LPS-initiated" mitogenesis of C3H/HeJ spleen cells. J Exp Med 144, 840-846.

Morrison, D. C., Vukajlovich, S. W., Ryan, J. L. \& Levin, J. (1987). Structural requirements for gelation of the Limulus amebocyte lysate by endotoxin. Prog Clin Biol Res 231, 55-73.

Nobile, C. J. \& Mitchell, A. P. (2007). Microbial biofilms: e pluribus unum. Curr Biol 17, R349-R353.

Odds, F. C. (1988). Candida and Candidosis, 2nd edn. London: Baillière Tindall.

O'Toole, G., Kaplan, H. B. \& Kolter, R. (2000). Biofilm formation as microbial development. Annu Rev Microbiol 54, 49-79.
Palma, C., Cassone, A., Serbousek, D., Pearson, C. A. \& Djeu, J. Y. (1992). Lactoferrin release and interleukin-1, interleukin-6, and tumor necrosis factor production by human polymorphonuclear cells stimulated by various lipopolysaccharides: relationship to growth inhibition of Candida albicans. Infect Immun 60, 4604-4611.

Pappas, P. G., Rex, J. H., Lee, J., Hamill, R. J., Larsen, R. A., Powderly, W., Kauffman, C. A., Hyslop, N., Mangino, J. E. \& other authors (2003). A prospective observational study of candidemia: epidemiology, therapy, and influences on mortality in hospitalized adult and pediatric patients. Clin Infect Dis 37, 634-643.

Peres-Bota, D., Rodriguez-Villalobos, H., Dimopoulos, G., Melot, C. \& Vincent, J. L. (2004). Potential risk factors for infection with Candida spp. in critically ill patients. Clin Microbiol Infect 10, 550555.

Pfaller, M. A. (1996). Nosocomial candidiasis: emerging species, reservoirs, and modes of transmission. Clin Infect Dis 22, S89-S94.

Pittet, D. \& Wenzel, R. P. (1995). Nosocomial bloodstream infections: secular trends in rates, mortality, and contribution to total hospital deaths. Arch Intern Med 155, 1177-1184.

Ramage, G., VandeWalle, K., Wickes, B. L. \& López-Ribot, J. L. (2001). Characteristics of biofilm formation by Candida albicans. Rev Iberoam Micol 18, 163-170.

Rangel-Frausto, M. S. (1999). The epidemiology of bacterial sepsis. Infect Dis Clin North Am 13, 299-312.

Rangel-Frausto, M. S., Wiblin, T., Blumberg, H. M., Saiman, L., Patterson, J., Rinaldi, M., Pfaller, M., Edwards, J. E., Jr, Jarvis, W. \& other authors (1999). National epidemiology of mycoses survey (NEMIS): variations in rates of bloodstream infections due to Candida species in seven surgical intensive care units and six neonatal intensive care units. Clin Infect Dis 29, 253-258.

Richards, M. J., Edwards, J. R., Culver, D. H. \& Gaynes, R. P. (1999). Nosocomial infections in medical intensive care units in the United States. Crit Care Med 27, 887-892.

Rietschel, E. T., Schade, U., Jensen, M., Wollenweber, H. W., Luderitz, O. \& Greisman, S. G. (1982). Bacterial endotoxins: chemical structure, biological activity and role in septicaemia. Scand J Infect Dis Suppl 31, 8-21.

Sabra, W., Lunsdorf, H. \& Zeng, A. P. (2003). Alterations in the formation of lipopolysaccharide and membrane vesicles on the surface of Pseudomonas aeruginosa PAO1 under oxygen stress conditions. Microbiology 149, 2789-2795.

Samaranayake, L. P. \& MacFarlane, T. W. (1990). Oral Candidosis. London: Wright.

Snydman, D. R. (2003). Shifting patterns in the epidemiology of nosocomial Candida infections. Chest 123, 500S-503S.

Tracey, K. J., Fong, Y., Hesse, D. G., Manogue, K. R., Lee, A. T., Kuo, G. C., Lowry, S. F. \& Cerami, A. (1987). Anti-cachectin/TNF monoclonal antibodies prevent septic shock during lethal bacteraemia. Nature 330, 662-664.

Vukajlovich, S. W. \& Morrison, D. C. (1985). Activation of murine spleen cells by lipid A: negative modulation of lipid A mitogenic activity by $\mathrm{O}$-antigen polysaccharide. J Immunol 135, 2546-2550.

Wisplinghoff, H., Bischoff, T., Tallent, S. M., Seifert, H., Wenzel, R. P. \& Edmond, M. B. (2004). Nosocomial bloodstream infections in US hospitals: analysis of 24,179 cases from a prospective nationwide surveillance study. Clin Infect Dis 39, 309-317.

Young, L. (1985). Gram negative sepsis. In Principles and Practice of Infectious Diseases, pp. 452-476. Edited by G. L. Mandell, R. G. Douglas \& J. E. Bennet. New York: Wiley. 